\title{
THE GENERALIZED FREDHOLM OPERATORS
}

BY

\section{KUNG-WEI YANG}

\begin{abstract}
Let $X, Y$ be Banach spaces over either the real field or the complex field. A continuous linear operator will be called a generalized Fredholm operator if $T(X)$ is closed in $Y$, and $\operatorname{Ker} T$ and Coker $T$ are reflexive Banach spaces. A theory similar to the classical Fredholm theory exists for the generalized Fredholm operators; and the similarity brings out the correspondence:

Reflexive Banach spaces $\longleftrightarrow$ finite-dimensional spaces, weakly compact operators $\longleftrightarrow$ compact operators, generalized Fredholm operators $\longleftrightarrow$ Fredholm operators, Tauberian operators with closed range $\longleftrightarrow$ semi-Fredholm operators.
\end{abstract}

1. Preliminaries. Let $k$ denote either the real field or the complex field. Let $B$ be the category whose objects are Banach spaces over $k$ and whose morphisms are continuous linear operators $T: X \rightarrow Y$. As usual, $B(X, Y)$ denotes the set of all continuous linear operators from $X$ to $Y$. With the norm $|T|=$ $\sup _{|x| \leqslant 1}|T(x)|, B(X, Y)$ becomes a Banach space over $k$.

We let $B(X, k)=X^{*}$, and $B(T, k)=T^{*} . I_{X}$ denotes the identity operator on $X$.

The sequence of continuous linear operators

$$
X \stackrel{S}{\longrightarrow} Y \stackrel{T}{\longrightarrow} Z
$$

is said to be exact at $Y$ if $S(X)=\operatorname{Ker} T$. The sequence of continuous linear operators

$$
X_{1} \stackrel{T_{1}}{\longrightarrow} X_{2} \stackrel{T_{2}}{\longrightarrow} X_{3} \rightarrow \cdots \rightarrow X_{n} \stackrel{T_{n}}{\longrightarrow} X_{n+1}
$$

is exact if it is exact at each $X_{i}(i=2,3, \ldots, n)$.

An exact sequence of the type $0 \rightarrow X \rightarrow Y \rightarrow Z \rightarrow 0$ is called a short exact sequence in $B$; by the open mapping theorem, there exists a closed linear subspace $Y_{1}$ of $Y$ such that $X \cong Y_{1}$ and $Z \cong Y / Y_{1}$, where $\cong$ denotes (and will denote) a topological isomorphism.

Exact sequences and diagram lemmas are the main tools employed in this

Received by the editors January 10, 1975 and, in revised form, February 28, 1975.

AMS (MOS) subject classifications (1970). Primary 47B30, 18A99.

Key words and phrases. Generalized Fredholm operator, reflexive Banach space, weakly compact operator, Tauberian operator, exact sequence. 
study of Banach spaces. By using these tools, we are also able to give some of the known theorems concerning reflexive Banach spaces and weakly compact operators new and more transparent proofs.

2. Exact sequences and diagram lemmas.

(2.1) ThEOREM. If $0 \rightarrow X \rightarrow Y \rightarrow Z \rightarrow 0$ is a short exact sequence in $B$, then $0 \rightarrow Z^{*} \rightarrow Y^{*} \rightarrow X^{*} \rightarrow 0$ is an exact sequence in $B$ [12].

Proof. The exactness at $Z^{*}, Y^{*}$ is clear. The exactness at $X^{*}$ is a consequence of the Hahn-Banach Theorem. Q.E.D.

(2.2) THEOREM. If $T \in B(X, Y)$ has a closed range, then

(i) $\operatorname{Ker} T^{*} \cong(\operatorname{Coker} T)^{*}$,

(ii) Coker $T^{*} \cong(\operatorname{Ker} T)^{*}$,

[8, Corollary 2, p. 111].

Proof. If $T \in B(X, Y)$ has a closed range, then Coker $T$ is well-defined and

$$
0 \rightarrow \operatorname{Ker} T \rightarrow X \stackrel{T}{\rightarrow} Y \rightarrow \text { Coker } T \rightarrow 0
$$

is an exact sequence in $B$. By Theorem (2.1), the sequence

$$
0 \rightarrow(\text { Coker } T)^{*} \rightarrow Y^{*} \stackrel{T^{*}}{\longrightarrow} X^{*} \rightarrow(\operatorname{Ker} T)^{*} \rightarrow 0
$$

is exact. Hence by the open mapping theorem,

$$
\operatorname{Ker} T^{*} \cong(\operatorname{Coker} T)^{*}, \quad \operatorname{Coker} T^{*} \cong(\operatorname{Ker} T)^{*} \text {. Q.E.D. }
$$

For any $X$ in $B$, there is a natural injection $n_{X}: X \rightarrow X^{* *}$ defined by $\left(n_{X}(x)\right)\left(x^{*}\right)=x^{*}(x)$ for all $x \in X$ and $x^{*} \in X^{*}$. As usual, we shall identify $X$ with $n_{X}(X)$ and omit the mention of $n_{X}$ when it is convenient to do so. Let $\bar{X}=X^{* *} / X$. If $T \in B(X, Y)$ then $\bar{T} \in B(\bar{X}, \bar{Y})$ is defined uniquely by the commutative diagram:

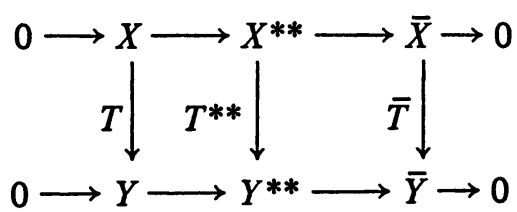

(2.3) THEOREM. $\quad-: B \rightarrow B$ is a covariant contracting functor, i.e.

(i) $\bar{I}_{X}=I_{\bar{X}}$;

(ii) if $S \in B(X, Y)$ and $T \in B(Y, Z)$, then $\overline{T S}=\bar{T} \bar{S}$;

(iii) $|\bar{T}| \leqslant|T|$.

Proof. (i) and (ii) are clear, (iii) follows from the fact that $\left|T^{* *}\right|=|T|$ and $\bar{T}$ is induced by $T^{* *}$. Q.E.D. 
(2.4) Theorem. For any $a, b$ in the field $k$, and any $S, T$ in $B(X, Y)$, $\overline{a S+b T}=a \bar{S}+b \bar{T}$.

Proof. Routine verification.

(2.5) Theorem. If $0 \rightarrow X \rightarrow Y \rightarrow Z \rightarrow 0$ is a short exact sequence in $B$, then $0 \rightarrow \bar{X} \rightarrow \bar{Y} \rightarrow \bar{Z} \rightarrow 0$ is exact.

Proof. Applying Theorem (2.1) twice, we see that

$$
0 \rightarrow X^{* *} \rightarrow Y^{* *} \rightarrow Z^{* *} \rightarrow 0
$$

is exact. Fitting these exact sequences together with the natural injections $X \rightarrow$ $X^{* *}, Y \rightarrow Y^{* *}, Z \rightarrow Z^{* *}$, we obtain the commutative diagram:

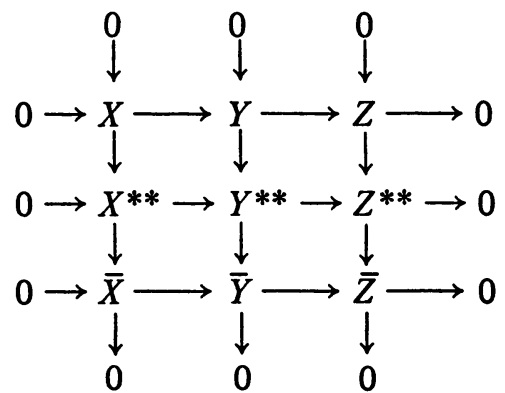

All the columns and all the rows in this commutative diagram are exact. The exactness of the last row is verified by "diagram chasing". So $0 \rightarrow \bar{X} \rightarrow \bar{Y} \rightarrow$ $\bar{Z} \rightarrow 0$ is indeed exact [12]. Q.E.D.

Theorems (2.3), (2.4) and (2.5) together show that $-: B \rightarrow B$ is a covariant linear contracting exact functor. As a corollary, we have

(2.6) Corollary. For any $T \in B(X, Y)$ with closed range,

(i) $\operatorname{Ker} \bar{T} \cong(\overline{\operatorname{Ker} T)}$,

(ii) Coker $\bar{T} \cong(\overline{\operatorname{Coker} T})$.

(Note: $\bar{T}$ does have a closed range.)

Proof. The sequence $0 \rightarrow \operatorname{Ker} T \rightarrow X \stackrel{T}{\longrightarrow} Y \rightarrow$ Coker $T \rightarrow 0$ is exact in $B$. By Theorem (2.5),

$$
0 \rightarrow \overline{(\operatorname{Ker} T)} \rightarrow \bar{X} \stackrel{\bar{T}}{\rightarrow} \bar{Y} \rightarrow(\overline{\operatorname{Coker} T}) \rightarrow 0
$$

is exact. By the open mapping theorem,

$\operatorname{Ker} \bar{T} \cong(\overline{\operatorname{Ker} T})$ and Coker $\bar{T} \cong(\overline{\operatorname{Coker} T})$. Q.E.D.

(2.7) THEOREM. If $S \in B(X, Y), T \in B(Y, Z)$ and $T S$ are range closed, then the following sequence of Banach spaces is exact [14]:

$0 \rightarrow \operatorname{Ker} S \rightarrow \operatorname{Ker} T S \rightarrow \operatorname{Ker} T \rightarrow$ Coker $S \rightarrow$ Coker $T S \rightarrow$ Coker $T \rightarrow 0$. 
Proof. The stated sequence comes from the following commutative diagram:

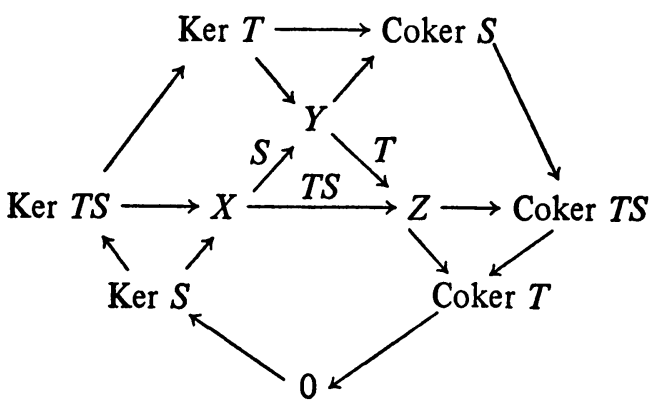

and it is utterly routine to verify that the sequence is exact. Q.E.D.

(2.8) THEOREM. If $T \in B(X, Y)$, then there exist topological isomorphisms $P, Q$ such that $(\bar{T})^{*}=P^{-1}\left(\overline{T^{*}}\right) Q$.

Proof. Consider the following two commutative diagrams with exact rows:

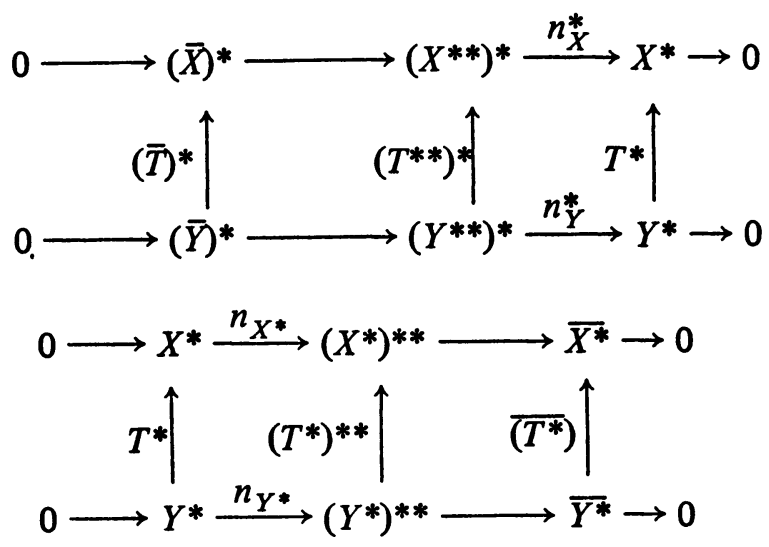

Since $n_{X}^{*} n_{X^{*}}=I_{X^{*}}$ and $n_{Y}^{*} n_{Y^{*}}=I_{Y^{*}}$, we have the following commutative diagram:

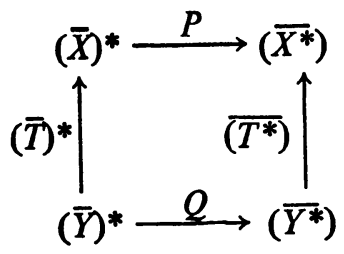

where $P$ and $Q$ are isomorphisms. Clearly, $(\bar{T})^{*}=P^{-1}\left(\overline{T^{*}}\right) Q$. Q.E.D.

(2.9) Theorem (SNAKe lemma For BANACH SPACES). If $T \in B\left(X, X_{1}\right)$, $U \in B\left(Y, Y_{1}\right), V \in B\left(Z, Z_{1}\right)$ all have closed ranges, and if the following commutative diagram in $B$ 


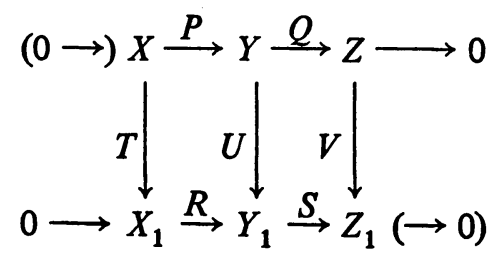

has exact rows, then there is a (natural) continuous linear operator $d$ such that $(0 \rightarrow)$ Ker $T \rightarrow \operatorname{Ker} U \rightarrow \operatorname{Ker} V \stackrel{d}{\longrightarrow}$ Coker $T \rightarrow$ Coker $U \rightarrow$ Coker $V(\rightarrow 0)$ is exact. (The data in parenthesis are supposed to occur in the conclusion if their counterparts are present in the hypothesis.) [1, Proposition (4.7), p. 26].

Proof. The definition of the operator $d$ is exactly the same as the usual abelian category situation [7, p. 203]: We consider the commutative diagram:

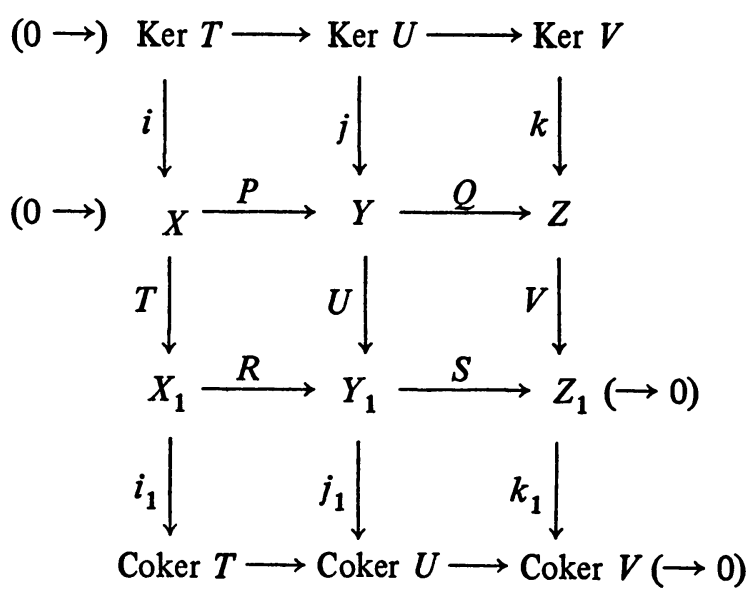

Choose any $z \in \operatorname{Ker} V . \quad k(z) \in Z$. $Q$ is onto. So there is some $y \in Y$ such that $Q(y)=k(z) . \quad U(y)$ has the property: $S U(y)=V Q(y)=V k(z)=0$. Hence there exists an $x \in X_{1}$ such that $R(x)=U(y) . d(z)$ is defined to be $i_{1}(x)$. It is easy to check that the definition is unambiguous and that the operator $d$ is linear. Applying [5, Lemma 1, p. 487] to the operators with closed range $Q$ and $R$, and observing that $k, U$ and $i_{1}$ are bounded, we can find constants $C_{1}, C_{2}, C_{3}, C_{4}$, $C_{5}$ such that:

1. For any $z \in \operatorname{Ker} V,|k(z)| \leqslant C_{1}|z|$.

2. For $k(z) \in Z$, there exists $y \in Y$ such that $Q(y)=k(z)$ and $|y| \leqslant$ $C_{2}|k(z)|$.

3. For $y \in Y,|U(y)| \leqslant C_{3}|y|$.

4. For $U(y)$ defined above, there exists some $x \in X_{1}$ such that $R(x)=$ $U(y)$ and $|x| \leqslant C_{4}|U(y)|$. 
5. For $x \in X_{1},\left|i_{1}(x)\right| \leqslant C_{5}|x|$.

Hence, there exists a constant $C$ such that for any $z \in \operatorname{Ker}(V),|d(z)| \leqslant C|z|$.

Thus $d$ is continuous. The exactness of the sequence

$(0 \rightarrow) \operatorname{Ker} T \rightarrow \operatorname{Ker} U \rightarrow \operatorname{Ker} V \rightarrow \operatorname{Coker} T \rightarrow \operatorname{Coker} U \rightarrow \operatorname{Coker} V(\rightarrow 0)$ is verified by the usual method [7, p. 203]. Q.E.D.

3. Reflexive Banach spaces. A Banach space $X$ is reflexive if the natural injection $n_{X}: X \rightarrow X^{* *}$ is an isomorphism (onto). Hence

(3.1) $X$ is reflexive $\Leftrightarrow \bar{X}=0 \Leftrightarrow \bar{I}_{X}=I_{\bar{X}}=0$.

(3.2) ThEOREM. If $X$ is a closed subspace of the Banach space $Y$ and if $Z=Y / X$, then $Y$ is reflexive if and only if $X$ and $Z$ are reflexive $[5$, Exercises 19, 20, p. 72].

Proof. $0 \rightarrow X \rightarrow Y \rightarrow Z \rightarrow 0$ is exact. Now by Theorem (2.5), $0 \rightarrow$ $\bar{X} \rightarrow \bar{Y} \rightarrow \bar{Z} \rightarrow 0$ is exact, whence $\bar{Y}=0 \Leftrightarrow \bar{X}=0$ and $\bar{Z}=0$. Q.E.D.

(3.3) Theorem. Let $X$ be a Banach space. Then $X$ is reflexive if and only if $X^{*}$ is reflexive [5, Corollary $\left.24, p .67\right]$.

Proof. $X$ is reflexive $\Leftrightarrow \bar{I}_{X}=0 \Leftrightarrow$ (by Theorem (2.8)) $\bar{I}_{X^{*}}=0 \Leftrightarrow X^{*}$ is reflexive. Q.E.D.

4. Weakly compact operators. Let $T: X \rightarrow Y$ be a continuous linear operator, and let $D$ be the closed unit sphere in $X$. T is weakly compact if the weak closure of $T(D)$ is compact in the weak topology of $Y$.

(4.1) THEOREM. The continuous linear operator $T: X \rightarrow Y$ is weakly compact if and only if $\bar{T}=0$.

Proof. See [5, Theorem 2, p. 482].

Clearly, we have

(4.2) CoRollary. If either $X$ or $Y$ is reflexive, then every $T \in B(X, Y)$ is weakly compact $[5$, Corollary $3, p .483]$.

Since the set of weakly compact operators is the kernel of the bar map (4.1) which has the properties of (2.3) and (2.4), it is a norm-closed linear subspace which is a "two-sided ideal" in the sense of $[9$, p. 17]

(4.3) TheOREM (GANTMACHER). An operator $T \in B(X, Y)$ is weakly compact if and only if $T^{*}$ is weakly compact.

Proof. Use Theorems (2.8) and (4.1). Q.E.D.

(4.4) Theorem ([5, Theorem 7, p. 425], $[3$, Theorem 1, p. 69]). $A$ 
Banach space $X$ is reflexive if and only if its closed unit sphere $D$ is compact in the weak topology.

Proof. $X$ is reflexive $\Leftrightarrow \bar{I}_{X}=0 \Leftrightarrow I_{X}$ is weakly compact $\Leftrightarrow I_{X}(D)=D$ is weakly compact. Q.E.D.

(Note, however, that the neatness of the proof is illusory. This was pointed out by the referee.)

This theorem corresponds to the theorem of F. Riesz which states that a Banach space $X$ is finite dimensional if and only if its closed unit sphere is compact in the norm topology.

(4.5) THEOREM. Let $T \in B(X, Y)$ be an operator with a closed range. Then, $T$ is weakly compact $\Leftrightarrow T(X)$ is reflexive.

Proof. $(\Leftrightarrow)$ Clear.

$\Leftrightarrow$ ) Without loss of generality, we may assume $T$ is onto. Then $\bar{T}$ is onto (Theorem (2.5)). But $\bar{T}=0$. Hence $\bar{Y}=0$. Q.E.D.

(The proof of this theorem was greatly simplified by a suggestion of the referee.)

5. Generalized Fredholm operators. We say that an operator $T \in B(X, Y)$ is generalized Fredholm if $T(X)$ is closed in $Y$, and $\operatorname{Ker} T$ and Coker $T$ are reflexive.

(5.1) THEOREM. If $T \in B(X, Y)$ is range closed, then $T$ is generalized Fredholm $\Leftrightarrow \bar{T}$ is an isomorphism (invertible).

Proof. $T$ is generalized Fredholm,

$\Leftrightarrow \overline{\operatorname{Ker} T}=0$ and $\overline{\operatorname{Coker} T}=0$.

$\Leftrightarrow$ (Corollary (2.6)) Ker $\bar{T}=0$, Coker $\bar{T}=0$.

$\Leftrightarrow \bar{T}$ is an isomorphism. Q.E.D.

(5.2) Theorem. Let $S \in B(X, Y), T \in B(Y, Z)$ and $T S$ be range closed. Then the sequence of Banach spaces

$$
0 \rightarrow \overline{\operatorname{Ker} S} \rightarrow \overline{\operatorname{Ker} T S} \rightarrow \overline{\operatorname{Ker} T} \rightarrow \overline{\operatorname{Coker} S} \rightarrow \overline{\operatorname{Coker} T S} \rightarrow \overline{\operatorname{Coker} T} \rightarrow 0
$$

is exact.

Proof. By Theorem (2.7),

$$
0 \rightarrow \operatorname{Ker} S \rightarrow \operatorname{Ker} T S \rightarrow \operatorname{Ker} T \rightarrow \operatorname{Coker} S \rightarrow \text { Coker } T S \rightarrow \text { Coker } T \rightarrow 0
$$

is exact. By Theorem (2.5),

$$
0 \rightarrow \overline{\operatorname{Ker} S} \rightarrow \overline{\operatorname{Ker} T S} \rightarrow \overline{\operatorname{Ker} T} \rightarrow \overline{\operatorname{Coker} S} \rightarrow \overline{\operatorname{Coker} T S} \rightarrow \overline{\operatorname{Coker} T} \rightarrow 0
$$

is exact. Q.E.D. 
The following theorems $((5.3)-(5.5))$ are immediate consequences of Theorem (5.2).

(5.3) Theorem. If $S \in B(X, Y)$ and $T \in B(Y, Z)$ are generalized Fredholm and TS has a closed range, then TS is generalized Fredholm.

(5.4) ThEOREM. Suppose $S \in B(X, Y)$ and $T \in B(Y, Z)$ are range closed, and suppose $T S \in B(X, Z)$ is generalized Fredholm. Then,

(i) $S$ is generalized Fredholm $\Leftrightarrow T$ is generalized Fredholm;

(ii) if $\operatorname{Ker} T$ is reflexive, then both $S$ and $T$ are generalized Fredholm;

(iii) if Coker $S$ is reflexive, then both $S$ and $T$ are generalized Fredholm.

(5.5) TheOREM. Let $T \in B(X, Y)$ have a closed range. If there exist $S$, $S^{\prime} \in B(Y, X)$ with closed ranges such that $S T$ and $T S^{\prime}$ are generalized Fredholm, then $T$ is generalized Fredholm.

(5.6) Theorem. Let $T \in B(X, Y)$ be range closed. Then, $T$ is generalized Fredholm $\Leftrightarrow T^{*}$ is generalized Fredholm.

Proof. $T$ is generalized Fredholm

$\Leftrightarrow$ (Theorem (5.1)) $\bar{T}$ is an isomorphism.

$\Leftrightarrow(\bar{T})^{*}$ is an isomorphism.

$\Leftrightarrow$ (Theorem (2.8)) $\left(\overline{T^{*}}\right)$ is an isomorphism.

$\Leftrightarrow T^{*}$ is generalized Fredholm. Q.E.D.

(5.7) THEOREM. Let $T \in B(X, Y)$ be a generalized Fredholm operator and $K \in B(X, Y)$ be a weakly compact operator such that $T+K$ has a closed range. Then $T+K$ is a generalized Fredholm operator.

Proof. By assumption, $T+K$ has a closed range. $\overline{T+K}=\bar{T}+\bar{K}=\bar{T}$ is an isomorphism. Hence by Theorem (5.1), $T+K$ is generalized Fredholm. Q.E.D.

(5.8) Theorem. If $T \in B\left(X, X_{1}\right), U \in B\left(Y, Y_{1}\right), V \in B\left(Z, Z_{1}\right)$ all have closed ranges and if the following commutative diagram in $B$ has exact rows

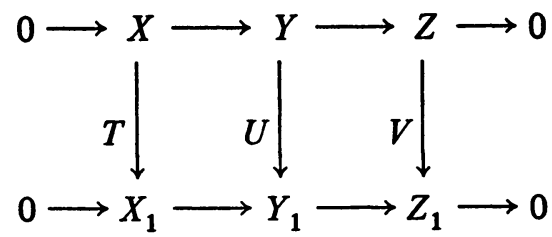

then the following sequence

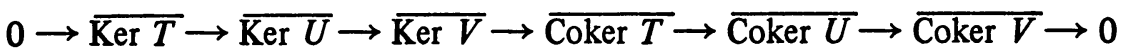
is exact. 
Proof. Use Theorems (2.9) and (2.5). Q.E.D.

An immediate consequence is:

(5.9) Theorem. Assumption as in Theorem (5.8). If any two of the operators $T, U, V$ are generalized Fredholm, then the third one is also generalized Fredholm.

(5.10) THEOREM. Let $X$ be a Banach space with the property that every closed reflexive subspace admits a closed complementary subspace. Let $Y$ be a Banach space with the property that every closed subspace with reflexive quotient space admits a closed complementary subspace. Then, $T \in B(X, Y)$ is a generalized Fredholm operator $\Leftrightarrow T$ is invertible modulo the weakly compact operators, and range closed.

Proof. $(\Rightarrow)$. Let $T$ be a generalized Fredholm operator. By definition, Ker $T$ is reflexive and $T(X)$ is closed in $Y$ and $Y / T(X)$ is reflexive. By the assumptions on $X$ and $Y$, there exist closed subspaces $U, V$ of $X$ and $Y$ respectively, such that $X \cong \operatorname{Ker} T \oplus U$, and $Y \cong T(X) \oplus V$. Clearly, $T \mid U: U \rightarrow T(X)$ is continuous and bijective. By the open mapping theorem, continuous inverse $(T \mid U)^{-1}$ exists. Identifying $Y$ and $T(X) \oplus V$, we may represent any $y \in Y$ as $y=y_{1}+y_{2}$, where $y_{1} \in T(X)$ and $y_{2} \in V$. Define $S: Y \rightarrow X$ by $S(y)=S\left(y_{1}+y_{2}\right)=$ $(T \mid U)^{-1} y_{1}$. Clearly, $S \in B(Y, X)$. It is easy to see that $I_{X}-S T=K$ is the projection of $X$ onto $\operatorname{Ker} T$ along $U$ and $I_{Y}-T S=L$ is the projection of $Y$ onto $V$ along $T(X)$. Since $\operatorname{Ker} T$ and $V$ are both reflexive, $K$ and $L$ are both weakly compact (Theorem (4.5)). This shows that $T$ is invertible modulo the weakly compact operators.

$\Leftrightarrow)$. Let $T$ be invertible modulo the weakly compact operators. Then there exist $S, S^{\prime} \in B(Y, X)$ such that $S T=I_{X}+K$ and $T S^{\prime}=I_{Y}+L$, where $K$ and $L$ are both weakly compact. Now,

$$
\bar{S} \bar{T}=\overline{S T}=\overline{I_{X}+K}=\bar{I}_{X}, \quad \bar{T} \bar{S}^{\prime}=\overline{T S^{\prime}}=\overline{I_{Y}+L}=\bar{I}_{Y} .
$$

This means $\bar{T}$ is invertible. By Theorem (5.1), $T$ is generalized Fredholm. Q.E.D.

Let $B(X)=B(X, X)$ and $W K(X) \subset B(X)$ be the closed two-sided ideal of weakly compact operators in $B(X)$. of $B(\bar{X})$.

(5.11) THEOREM. $B(X) / W K(X)$ is isomorphic to a topological $k$-subalgebra

Proof. By Theorems (2.3), (2.4) and (4.1), $T+W K(X) \mapsto \bar{T}$ is a topological monomorphism. Q.E.D.

In view of the above results, it is probably appropriate to call the algebra $B(X) / W K(X)$, the generalized Calkin algebra. (Compare [15, p. 127] .) 
6. Tauberian and co-Tauberian operators. Let $T \in B(X, Y) . T$ is called a Tauberian operator if $T^{* *-1}\left(n_{Y}(Y)\right) \subset n_{X}(X)$ [6]. Equivalently: $T$ is Tauberian if and if $\operatorname{Ker} \bar{T}=0$.

(6.1) THEOREM [6]. If $T \in B(X, Y)$ has a closed range, then $T$ is Tauberian if and only if $\operatorname{Ker} T$ is reflexive.

Proof. Since $T$ has a closed range, we may apply Corollary (2.6) and obtain: $\operatorname{Ker}(\bar{T})=\overline{\operatorname{Ker} T}$. Clearly, $T$ is Tauberian $\Leftrightarrow \operatorname{Ker}(\bar{T})=0 \Leftrightarrow \overline{\operatorname{Ker} T}=0 \Leftrightarrow$ Ker $T$ is reflexive. Q.E.D.

In view of Theorem (6.1), we see that the classical notion corresponding to a Tauberian operator with a closed range is that of a semi-Fredholm operator $[11$, p. 125].

(6.2) THEOREM. If $T \in B(X, Y)$ is Tauberian and $K \in B(X, Y)$ is weakly compact, then $T+K$ is Tauberian.

ProOF. $\operatorname{Ker}(\overline{T+K})=\operatorname{Ker}(\bar{T}+\bar{K})=\operatorname{Ker}(\bar{T})=0$. Hence, $T+K$ is Tauberian. Q.E.D.

(6.3) THEOREM. If $S \in B(X, Y)$ and $T \in B(Y, Z)$ are Tauberian, then TS is Tauberian [6].

Proof. It is easy to see that $0 \rightarrow \operatorname{Ker} \bar{S} \rightarrow \operatorname{Ker} \bar{T} \bar{S} \rightarrow \operatorname{Ker} \bar{T}$ is exact. So, $S$ and $T$ are Tauberian $\Rightarrow \operatorname{Ker} \bar{S}=0$ and $\operatorname{Ker} \bar{T}=0 \Rightarrow \operatorname{Ker} \overline{T S}=\operatorname{Ker} \overline{T S}=0$ $\Rightarrow T S$ is Tauberian. Q.E.D.

Let $T \in B(X, Y) . T$ is called a co-Tauberian operator if $T$ has a closed range and $\operatorname{Coker}(\bar{T})=0$. (Note that if $T$ has a closed range, then $\bar{T}$ also has a closed range.)

(6.4) THEOREM. If $T \in B(X, Y)$ has a closed range then $T$ is co-Tauberian if and only if Coker $T$ is reflexive.

Proof. $T$ is by assumption range closed. So, $T$ is co-Tauberian $\Leftrightarrow$ $\operatorname{Coker}(\bar{T})=0 \Leftrightarrow($ Corollary (2.6)) $\overline{\operatorname{Coker} T}=0 \Leftrightarrow$ Coker $T$ is reflexive. Q.E.D.

(6.5) THEOREM. If $T \in B(X, Y)$ is co-Tauberian, $K \in B(X, Y)$ is weakly compact, and $T+K$ has a closed range, then $T+K$ is co-Tauberian.

Proof. $\operatorname{Coker}(\overline{T+K})=\operatorname{Coker}(\bar{T}+\bar{K})=\operatorname{Coker}(\bar{T})=0$. So $T+K$ is co-Tauberian. Q.E.D.

(6.6) ThEOREM. If $S \in B(X, Y)$ and $T \in B(Y, Z)$ are co-Tauberian, and TS has a closed range, then TS is co-Tauberian.

Proof. The following is an exact sequence in $B$ : 


$$
\operatorname{Coker}(\bar{S}) \rightarrow \operatorname{Coker}(\bar{T} \bar{S}) \rightarrow \operatorname{Coker}(\bar{T}) \rightarrow 0 .
$$

Now, $S$ and $T$ are co-Tauberian $\Rightarrow \operatorname{Coker}(\bar{S})=0$ and $\operatorname{Coker}(\bar{T})=0 \Rightarrow \operatorname{Coker}(\overline{T S})$ $=\operatorname{Coker}(\bar{T} \bar{S})=0 \Rightarrow T S$ is co-Tauberian. Q.E.D.

(6.7) ThEOREM. Let $T \in B(X, Y)$. Then, $T$ is generalized Fredholm $\Leftrightarrow T$ is Tauberian and co-Tauberian.

Proof. Use Theorems (6.1) and (6.4). Q.E.D.

(6.8) THEOREM. Same assumption as in Theorem (5.8). If $U$ is generalized Fredholm, then $T$ is Tauberian and $V$ is co-Tauberian.

Proof. Use Theorems (5.8), (6.1) and (6.4). Q.E.D.

(6.9) THEOREM. If $T \in B(X, Y)$ is range closed, then

(i) $T$ is Tauberian $\Leftrightarrow T^{*}$ is co-Tauberian,

(ii) $T$ is co-Tauberian $\Leftrightarrow T^{*}$ is Tauberian.

Proof. (i) Since $\bar{T}$ also has a closed range, we may apply Theorem (2.2) and obtain $(\operatorname{Ker} \bar{T})^{*} \cong \operatorname{Coker}(\bar{T})^{*}$. By Theorem (2.8), there exist topological isomorphisms $P, Q$ such that $(\bar{T})^{*}=P^{-1}\left(\overline{T^{*}}\right) Q$. Hence, $(\operatorname{Ker} \bar{T})^{*} \cong$ $\operatorname{Coker}\left(P^{-1}\left(\overline{T^{*}}\right) Q\right)$.

Now, $T$ is Tauberian $\Leftrightarrow \operatorname{Ker} \bar{T}=0$

$\Leftrightarrow \operatorname{Coker}\left(P^{-1}\left(T^{*}\right) Q\right)=0$

$\Leftrightarrow \operatorname{Coker}\left(\overline{T^{*}}\right)=0$

$\Leftrightarrow T^{*}$ is co-Tauberian.

The proof of (ii) is similar to that of (i). Q.E.D.

7. Examples of generalized Fredholm operators. Generalized Fredholm operators can be constructed from Fredholm operators by tensoring.

First, a few remarks on split exact sequences and topological tensor products.

The short exact sequence in $B, 0 \rightarrow X \stackrel{S}{\longrightarrow} Y \stackrel{T}{\longrightarrow} Z \rightarrow 0$, is a split exact sequence if either there exists a $U \in B(Y, X)$ such that $U S=I_{X}$, or there exists a $V \in B(Z, Y)$ such that $T V=I_{Z}$. If the above short exact sequence is split, then $Y \cong X \oplus Z$. In fact, in that case $Y=S(X) \oplus(\operatorname{Ker} U)$, and $T$ restricted to $\operatorname{Ker} U \rightarrow Z$ is a topological isomorphism. It is, therefore, clear that if $0 \rightarrow X$ $\stackrel{S}{\longrightarrow} Y \stackrel{T}{\longrightarrow} Z \rightarrow 0$ is a split exact sequence, then for any Banach space $H$, the sequence

$$
0 \rightarrow X \hat{\otimes} H \stackrel{S \hat{\otimes} I_{H}}{\longrightarrow} Y \hat{\otimes} H \stackrel{T \hat{\otimes} I_{H}}{\longrightarrow} Z \hat{\otimes} H \rightarrow 0
$$

is exact. Here, $X \hat{\otimes} H$ denotes the topological tensor product completed with respect to the greatest crossnorm of Schatten $[10$, p. 36]

Now, let $T \in B(X, Y)$ be a Fredholm operator. Then, [8, Theorem $6, \mathrm{p}$. 
109 and Theorem 1, p. 119]

$0 \rightarrow \operatorname{Ker} T \rightarrow X \rightarrow T(X) \rightarrow 0$ and $0 \rightarrow T(X) \rightarrow Y \rightarrow$ Coker $T \rightarrow 0$ are both split exact sequences. Let $H$ be a reflexive Banach space. We see that

$$
0 \rightarrow \operatorname{Ker} T \hat{\otimes} H \rightarrow X \hat{\otimes} H \stackrel{T \hat{\otimes} I_{H}}{\longrightarrow} Y \hat{\otimes} H \rightarrow \text { Coker } T \hat{\otimes} H \rightarrow 0
$$

is an exact sequence. Since $\operatorname{Ker} T \hat{\otimes} H$ and Coker $T \hat{\otimes} H$ are reflexive, $T \hat{\otimes} I_{H}$ is a generalized Fredholm operator.

8. Problems and comments.

(8.1) Can an index theory be developed for the generalized Fredholm operators?

(8.2) Further investigation into the correspondence stated in the abstract may lead to fruitful results.

(8.3) Many of the theorems on the generalized Fredholm operators may be extended to the following more general operators:

(i) operator $T \in B(X, Y)$ such that $T(X)$ is closed in $Y$, and $\overline{\operatorname{Ker} T}$ and $\overline{\text { Coker } T}$ are finite dimensional (i.e. $\operatorname{Ker} T$ and Coker $T$ are quasi-reflexive in the sense of [2]);

(ii) operator $T \in B(X, Y)$ such that $T(X)$ is closed in $Y$ and $\overline{\overline{\operatorname{Ker} T}}=0$ and $\overline{\overline{\text { Coker } T}}=0$; etc.

(8.4) Other applications of exact sequences in elementary functional analysis exist [13].

Appendix. In this appendix, we shall prove many of the standard theorems in the classical Fredholm theory by using the results in \$2. Recall that an operator $T \in B(X, Y)$ is called a Fredholm operator if $\operatorname{dim}(\operatorname{Ker} T)$ and $\operatorname{dim}(\operatorname{Coker} T)$ are finite. (Here Coker $T$ refers to the algebraic cokernel.) Notice that if $T$ is Fredholm, then $T(X)$ is closed in $Y$ [8, Theorem 1, p. 119]. The integer ind(T) $=\operatorname{dim}(\operatorname{Ker} T)-\operatorname{dim}($ Coker $T)$ is called the index of $T$.

The following results ((A.1)-(A.5)) follow immediately from Theorem (2.7) and the simple observation that if $A \rightarrow B \rightarrow C$ is exact at $B$, and if $\operatorname{dim} A$ and $\operatorname{dim} C$ are finite then $\operatorname{dim} B$ is finite.

(A.1) THEOREM. If $S \in B(X, Y)$ and $T \in B(Y, Z)$ are Fredholm, then $T S$ is Fredholm.

(A.2) THEOREM. Suppose $S \in B(X, Y)$ and $T \in B(Y, Z)$ are operators such that TS is Fredholm. Then

(i) $S$ is Fredholm $\Leftrightarrow T$ is Fredholm;

(ii) if $\operatorname{dim}(\operatorname{Ker} T)$ is finite, then both $S$ and $T$ are Fredholm;

(iii) if $\operatorname{dim}($ Coker $S$ ) is finite, then both $S$ and $T$ are Fredholm. 
(A.3) THEOREM. Let $T \in B(X, Y)$. If there are $S, S^{\prime} \in B(Y, X)$ such that $S T$ and $T S^{\prime}$ are Fredholm, then $T$ is Fredholm.

(A.4) Corollary. Let $T_{1}, \ldots, T_{n} \in B(X, X)$, which commute. Then, $T_{1} \cdots T_{n}$ is Fredholm $\Leftrightarrow T_{i}$ is Fredholm for $i=1, \ldots, n$.

(A.5) Corollary. If $T \in B(X, X)$ is such that $T^{n}$ is Fredholm for some $n \geqslant 1$, then $T$ is Fredholm.

(A.6) Theorem. If $S \in B(X, Y)$ and $T \in B(Y, Z)$ are Fredholm, then (TS is Fredholm and) $\operatorname{ind}(T S)=\operatorname{ind}(S)+\operatorname{ind}(T)$.

Proof. This identity follows from Theorem (2.7) and the fact that if $0 \rightarrow A_{0} \rightarrow A_{1} \rightarrow \cdots \rightarrow A_{n} \rightarrow 0$ is an exact sequence of finite-dimensional Banach spaces, then

$$
\sum_{i=0}^{n}(-1)^{i} \operatorname{dim}\left(A_{i}\right)=0 . \quad \text { Q.E.D. }
$$

(A.7) Theorem. If $T \in B(X, Y)$ is Fredholm, then $T^{*} \in B\left(Y^{*}, X^{*}\right)$ is Fredholm and $\operatorname{ind}\left(T^{*}\right)=-\operatorname{ind}(T)$.

PROOF. This follows from Theorem (2.2). Q.E.D.

(A.8) THEOREM. If $T \in(X, Y)$ is Fredholm, then $\operatorname{ind}(T)=\operatorname{dim}(\operatorname{Ker} T)-$ $\operatorname{dim}\left(\operatorname{Ker} T^{*}\right)$.

Proof. Theorem (2.2). Q.E.D.

(A.9) Theorem. Let $T \in B(X, Y)$. Then, $T$ is Fredholm $\Leftrightarrow T$ is invertible modulo the compact operators.

PROof. $\Leftrightarrow$ ) The proof of this implication is standard (see the corresponding part in [8, Theorem 2, p. 120]).

$\Leftrightarrow$ This follows from (A.3), (A.5) and [4, (11, 3.3), p. 321]. Q.E.D.

(A.10) THEOREM. If $T \in B(X, Y)$ is Fredholm and $K \in B(X, Y)$ is a compact operator, then $T+K$ is Fredholm, and ind $(T+K)=\operatorname{ind}(T)$.

Proof [11, p. 114]. By Theorem (A.9), there exist $S, S^{\prime} \in B(Y, X)$ such that $S T-I_{X}=K_{1}$ and $T S^{\prime}-I_{Y}=K_{2}$ are compact. Clearly,

$$
\begin{aligned}
& S(T+K)=I_{X}-K_{1}+S K=I_{X}-K_{3}, \\
& (T+K) S=I_{Y}-K_{2}+K S=I_{Y}-K_{4},
\end{aligned}
$$

and $K_{3}, K_{4}$ are compact operators, whence $T+K$ is a Fredholm operator. From the above equalities we have

$$
\operatorname{ind}(S)+\operatorname{ind}(T+K)=\operatorname{ind}\left(I_{X}-K_{3}\right)=0,
$$




$$
\operatorname{ind}(S)+\operatorname{ind}(T)=\operatorname{ind}\left(I_{X}-K_{1}\right)=0 .
$$

Hence $\operatorname{ind}(T+K)=\operatorname{ind}(T)$. Q.E.D.

(A.11) THEOREM. Same assumption as in Theorem (5.8).

If any two of the operators $T, U, V$ are Fredholm, then the third one is also Fredholm.

Proof. This follows immediately from Theorem (2.9). Q.E.D.

ACKNOWLedgements. I wish to thank Professor Albert Wilansky for much helpful correspondence, Professors William J. Davis and M. Mielke, each for pointing out an error in the second draft of this article, my teacher, Professor George Whaples, for his many contributions, and the referee for many useful comments.

\section{REFERENCES}

1. H. Bass, Algebraic K-theory, Benjamin, New York and Amsterdam, 1968. MR 40 \#2736.

2. P. Civin and B. Yood, Quasi-reflexive spaces, Proc. Amer. Math. Soc. 8 (1957), 906-911. MR 19, 756. 1973.

3. M. M. Day, Normed linear spaces, 3rd ed., Springer-Verlag, New York and Berlin,

4. J. A. Dieudonné, Fondements de l'analyse moderne, Pure and Appl. Math., vol. 10, Academic Press, New York and London, 1960. MR 22 \#11074.

5. N. Dunford and J. T. Schwartz, Linear operators. I. General theory, Pure and Appl. Math., vol. 7, Interscience, New York and London, 1958. MR 22 \#8302.

6. N. Kalton and A. Wilansky, Tauberian operators on Banach spaces, Preprint, 1974.

7. S. Mac Lane, Categories for the working mathematician, Springer-Verlag, New York and Berlin, 1971.

8. R. S. Palais, Seminar on the Atiyah-Singer index theorem, Ann. of Math. Studies, no. 57, Princeton Univ. Press, Princeton, N. J., 1965. MR 33 \#6649.

9. N. Popescu, Abelian categories with applications to rings and modules, Academic Press, London and New York, 1973.

10. R. Schatten, $A$ theory of cross-spaces, Ann. of Math. Studies, no. 26, Princeton Univ. Press, Princeton, N. J., 1950. MR 12, 186.

11. M. Schechter, Principles of functional analysis, Academic Press, New York and London, 1971.

12. K.-W. Yang, $A$ note on reflexive Banach spaces, Proc. Amer. Math. Soc. 18 (1967), 859-861. MR 35 \#5905.

13. - Completion of normed linear spaces, Proc. Amer. Math. Soc. 19 (1968),

801-806. MR 37 \#1962.

14. Index of Fredholm operators, Proc. Amer. Math. Soc. 41 (1973), 329-

330. MR 47 \#7492.

15. R. G. Douglas, Banach algebra techniques in operator theory, Academic Press, New York and London, 1972.

DEPARTMENT OF MATHEMATICS, WESTERN MICHIGAN UNIVERSITY, KALAMAZOO, MICHIGAN 49001 